\title{
Nuclear Electromagnetic Generator: Mathematical Model on Toroidal Vacuum Scheme
}

\author{
V. Yu. Tertychny-Dauri \\ Department of Physics and Engineering, Saint-Petersburg National Research University of Information Technologies, Mechanics \\ and Optics, Saint-Petersburg, Russia \\ Email: tertychny-dauri@mail.ru
}

How to cite this paper: Tertychny-Dauri, V.Yu. (2018) Nuclear Electromagnetic Generator: Mathematical Model on Toroidal Vacuum Scheme. Open Access Library Journal, 5: e4524.

https://doi.org/10.4236/oalib.1104524

Received: March 20, 2018

Accepted: April 16, 2018

Published: April 19, 2018

Copyright (c) 2018 by author and Open Access Library Inc.

This work is licensed under the Creative Commons Attribution International License (CC BY 4.0).

http://creativecommons.org/licenses/by/4.0/

\section{(c) (i) Open Access}

\begin{abstract}
This article be parenthetic to the cycle of works devoted mathematical description of analytical means for the solution of some problems of the nuclear electromagnetic generator functioning. These problems are connected with the parameters calculation of external electromagnetic field on the toroid and parameters of charged kinetics which products this field is induced. General scheme of action for the nuclear electromagnetic toroidal vacuum generator (in shortened form: nuclegen) with controlled shields from the radioactive fission substance is adduced. Shields may consist a several layers executed the role of reflector or moderator for oppositely charged products of the fission. Parameters calculation of induced electromagnetic field in the external winding of toroid for the different initial (driving) regimes of functioning generator is produced also.
\end{abstract}

\section{Subject Areas}

Theoretical Physics, Modern Physics, Nuclear Physics

\section{Keywords}

Electromagnetic Modelling, External and Internal Electromagnetic Fields

\section{Introduction}

Presented work is related to research of the generation phenomenon of increasing external electromagnetic energy attached to rapidly increasing velocity (density) of passing for internal process of the chain nuclear fission reaction. Cascaded development of the chain nuclear fission reaction leads then 
to the vigorous intensification of external induced electric current and electromagnetic field. Besides the scenario of development for directional high-speed motion of increasing numbers of charged products of the fission may serve a basis for the creation of superpowerful energetical nuclear generators and electromagnetic power designs and accelerators of varied sorts particles also (see works [1] [2] [3]).

Properly from the physics of considered process the nuclegens transform kinetic energy of charged products of the fission (splinters and anougher particles) immediately into electroenergy, as distinct from nuclear reactors, where only part of thermal energy of fission products transforms into electroenergy. It is clear that nuclegens according to own power potentials extremely powerfully will be surpassing analogous potentials of modern nuclear reactors.

Principled scheme of nuclear generator presents the vacuum toroid with uniformly wound external conductive spiral. Along all over ring the circular shields are installed from fissionable radioactive substance into internal hollow part of nuclegen. In the present case shields fulfil the role of fuel pins at usual nuclear reactor. In absence of moderator the controlled process of nuclear chain fission is realized at the expense of controlled turn of shields around own axes of revolution. Obviously that the change of shields position concerning the general neutron-fragmentation stream leads to the immediate change of the course of fission process.

Intervals between of shields are choosen from counting, firstly, of effectively increasing passing of the chain fission reaction and, secondly, of enough complete run of splinters and another fission products (within beginning of active recombination and neutralization of charges). Of course, it is possible and another just like engineering variants of concentration of fissionable substance into toroidal vacuum generator which lead to controlled intensification or weakening of the nuclear fission process.

Just one more comment. It concerns making of nuclear resonator for directional motion of charged particles. As a such resonator may serve in itself fuel shield if one of sides will be the reflector (the moderator) for positively charged fission splinters (particles), and another, opposite side-for negatively charged fission splinters (particles). Finally, it is possible variant with one reflector on boundary shield surface; variant when the voltage is given on surface of shield and so on.

Sections 2 - 5 are devoted to the description of characteristics of electromagnetic field for the toroidal nuclegen. In Section 2 Maxwell's equations are used for the notion of general motion picture of charged particles. In Section 3, the transformation of Maxwell's equations is realized for the forming wave equations. Section 4 is devoted to the description of fading regime of external electromagnetic field by the switching-off field sources. In Section 5, the calculation of parameters for electromagnetic fields is conducted. 


\section{General Description of Electromagnetic Process Maxwell's Equations}

Further for simple of text record we shall not be distinguish the vector and scalar values between of itself, so far as from the context of presented analytical expressions it is becoming clearly which value (vector or scalar) is meant. Suppose that on external winding of toroid the electric exterior current is given with amperage $I_{0}$ and density $j_{0}$, exciting directed electromagnetic field within of nuclear generator. We shall be characterizing this external field by means of strength $E_{0}$ and electric induction $D_{0}$ (for electric field), of strength $H_{0}$ and magnetic induction $B_{0}$ (for magnetic field). In considered case for the field at vacuum we have

$$
D_{0}=\varepsilon_{0} E_{0}, B_{0}=\mu_{0} H_{0},
$$

where $\varepsilon_{0}=8.854 \times 10^{-12} \approx(1 / 36 \pi) \times 10^{-9} \mathrm{~F} / \mathrm{m}$ (Farad/metre)-electric constant, $\mu_{0}=4 \pi \times 10^{-7}=1.257 \times 10^{-6} \mathrm{Hn} / \mathrm{m}$ (Henry/metre)-magnetic constant.

In a position when shields are closed and the process of chain fission is started the uncrowded intervals between them are filled with multitude of charged high-speed particles and fission splinters. In essence, enough strong external electromagnetic field $\left(E_{0}, D_{0}, H_{0}, B_{0}\right)$ with current $I_{0}$ is necessary only for their initial interdirected accelerated motion along toroid. Besides, induced in external winding the current $I_{*}$ and the electromagnetic field $\left(E_{*}, D_{*}, H_{*}, B_{*}\right)$ discover also (as it will be shown below) exponentional intensive avalance-like growth with growth of neutrons number and free charges under exponentional law. This is phenomenological aspect of a matter. We attemp to envelop foregoing in analitical expressions.

Make use of general macroscopic Maxwell's equations for variable electromagnetic field $(E, D, H, B)$ are written in differential (local) form:

$$
\begin{aligned}
& \operatorname{rot} E=-\frac{\partial B}{\partial t}, \operatorname{div} D=\rho, \\
& \operatorname{rot} H=j+\frac{\partial D}{\partial t}, \operatorname{div} B=0,
\end{aligned}
$$

with boundary conditions on $\Gamma$ from a certain region $\Omega$, namely $(E, D, H, B)_{\Gamma} \in \Omega$, where $j+\partial D / \partial t$ is the density of fully current, $j_{d}$ is the density of displacement current, $j$ is the density of conductivity current, $\rho$ is the volumetric charged density, $I=\int_{S} j \mathrm{~d} S$ is the conductivity current,

$I_{d}=\frac{\mathrm{d}}{\mathrm{d} t} \int_{S} D \mathrm{~d} S$ is the displacement current, $S$ is a surface. Owing to vector identity $\operatorname{div} \operatorname{rot} H=0$ we have $\operatorname{div}(j+\partial D / \partial t)=0$. If in this relation trade places operators $\operatorname{div}$ and $\partial / \partial t$ then taking into account $\operatorname{div} D=\rho$ we obtain the equation of continuity

$$
\operatorname{div} j=-\frac{\partial \rho}{\partial t}
$$

Note also the connection between $j$ and $E: j=\sigma E$, where $\sigma$ is specific 
conductivity. In general system of Maxwell's Equations (1) according to the principle of superposition we have the addition of external and internal electromagnetic fields:

$$
E=E_{0}+E_{*}, H=H_{0}+H_{*} .
$$

Suppose that as a result of chain nuclear fission reaction in the time moment $t$ in "free" toroidal volume there is some finite number $K$ of "free" charged particles and splinters (light and heavy) of different sign and $N$ neutrons. By interdirected longitudinal motion into external electromagnetic field of positive and negative particles there are created "superthin" currents of convective form with densities $j_{* i}=\rho_{i} v_{i}, i=\overline{1, K}$, where $\rho_{i}$ is volumetric density of charge allocation of $i$-th particle (splinter), $v_{i}=v_{i}(t)$ is its velocity. i.e. resultant (at the first approximation) current inside of nuclear toroidal generator is characterized by the density

$$
j_{*}=\sum_{i=1}^{K} j_{* i}=\sum_{i=1}^{K} \rho_{i} v_{i}
$$

It is necessary to add that on the each charged $i$-th particle is effected, as is known, the Lorenz's force into external electromagnetic field

$$
F_{i}=Z_{i} E+Z_{i}\left(v_{i} \times B\right)
$$

where $Z_{i}$ is charge of $i$-th particle (splinter), moreover according to Gaussian theorem for the volume $V$ with surface $S$ we have

$$
\oint D_{i} \mathrm{~d} S=Z_{i}=\int \rho_{i} \mathrm{~d} V, I_{i}=\frac{\mathrm{d} Z_{i}}{\mathrm{~d} t} .
$$

As a result of the action of Lorenz's force (3) the partiale trajectory inside of toroid will be tighten up in spiral. General picture motion of charged particles and another products of fission nuclear into powerful directed increasing electromagnetic field must present, apparently, longitudinal intertwisting with speed-up the spiral electromagnetic cord inside of toroidal nuclegen in itself.

\section{Electromagnetic Modelling Transformation of Maxwell's Equations}

Convert Maxwell's equations for vacuum (1) and use standard methods of the vector differential analysis trying to solve system (1) on magnetic $(H)$ and on electric $(E)$ components. We have from the second group of equations

$$
\frac{\operatorname{rot} H}{\sigma}=E+\frac{\varepsilon_{0}}{\sigma} \frac{\partial E}{\partial t}, \operatorname{div} H=0
$$

So the field $E$ is determined with the aid of Equation (4) owing to known $H$. Further for any vector $A$ we avail identities

$$
\operatorname{div} \operatorname{rot} A=0, \quad \operatorname{rotrot} A=\operatorname{grad} \operatorname{div} A-\Delta A,
$$

where $\Delta$ is Laplace's operator. We obtain from Equation (4) ( $\sigma=$ const ):

$$
\frac{\operatorname{rotrot} H}{\sigma}=\operatorname{rot} E+\frac{\varepsilon_{0}}{\sigma} \frac{\partial}{\partial t} \operatorname{rot} E .
$$


Substitute the expression for $\operatorname{rot} E$ from the first group of Equation (1) at Equation (6). Taking into account correlations (5) we have the wave equation for $H$ :

$$
\Delta H=\sigma \mu_{0} \frac{\partial H}{\partial t}+\varepsilon_{0} \mu_{0} \frac{\partial^{2} H}{\partial t^{2}},\left.\quad H\right|_{\Gamma} \in \Omega .
$$

Analogously we obtain the wave equation for $E$ :

$$
\Delta E-\operatorname{graddiv} E=\sigma \mu_{0} \frac{\partial E}{\partial t}+\varepsilon_{0} \mu_{0} \frac{\partial^{2} E}{\partial t^{2}},\left.\quad E\right|_{\Gamma} \in \Omega .
$$

\section{Fade Regime of External Field by the Switching-Off of Field Sources}

We should be considering that charged electromagnetic cord in toroid presents in the aggregate the conductor situated into electromagnetic field. Then by instantaneous switching-off of field sources the field would not disappear but it would be exponentionally in the time fading according to spatial-evolutionary Equations ((7) and (8)). Following to known solution methods of problems of mathematical physics we should be solutions of Equations ((7) and (8)) searching correspondingly in the form

$$
H=H_{m}(x, y, z) \mathrm{e}^{-\gamma_{m} t}, \quad E=E_{m}(x, y, z) \mathrm{e}^{-\delta_{m} t}
$$

with constant coefficients $\gamma_{m}, \delta_{m}, m=\overline{1, \infty}$; here $x, y, z$ are cartesian spatial variables, $t$ is the time. We obtain equations for vector functions $H_{m}(x, y, z), E_{m}(x, y, z)$ :

$$
\begin{gathered}
\Delta H_{m}=\left(-\sigma \mu_{0} \gamma_{m}+\varepsilon_{0} \mu_{0} \gamma_{m}^{2}\right) H_{m}, \\
\Delta E_{m}-\operatorname{grad} \operatorname{div} E_{m}=\left(-\sigma \mu_{0} \delta_{m}+\varepsilon_{0} \mu_{0} \delta_{m}^{2}\right) E_{m} .
\end{gathered}
$$

Equations ((10), (11)) have not equal to zero solutions satisfying boundary conditions under the certain set of eigenvalues $\gamma_{m}, \delta_{m}$.

Elucidate the character of alteration for numbers $\gamma_{m}, \delta_{m}$. Carry out this analysis as an example $\gamma_{m}$. Take Equation (10) in the form

$$
\operatorname{rot} \operatorname{rot} H_{m}=\left(\sigma \mu_{0} \gamma_{m}-\varepsilon_{0} \mu_{0} \gamma_{m}^{2}\right) H_{m}
$$

and scalar multiply vector-line $H_{m}^{*}$ on this equation (here ${ }^{*}$ above is the transposition sign). We obtain after integration over total space

$$
\left(\sigma \mu_{0} \gamma_{m}-\varepsilon_{0} \mu_{0} \gamma_{m}^{2}\right) \int\left\|H_{m}\right\|^{2} \mathrm{~d} V=\int H_{m}^{*} \operatorname{rot} \operatorname{rot} H_{m} \mathrm{~d} V=\int\left\|\operatorname{rot} H_{m}\right\|^{2} \mathrm{~d} V .
$$

Reality and positivity of value $\sigma \mu_{0} \gamma_{m}-\varepsilon_{0} \mu_{0} \gamma_{m}^{2}$ follow with evidence from equality (12). This condition answered numbers:

1) real $\gamma_{m}$, for which $0<\gamma_{m}<\sigma / \varepsilon_{0}$;

2) complex $\gamma_{m}=a_{m}+i b_{m}$, where $a_{m}=\sigma /\left(2 \varepsilon_{0}\right), i^{2}=-1, b_{m}$ are arbitrary real numbers.

Quite analogous opinions may be adduce for numbers $\delta_{m}$, if to proceed from Equation (11). In either case we have to deal with dynamic process which 
solutions (9) fading exponentionally in the time. At last, we set the distribution of field in initial time moment with the aid of functions $H^{0}(x, y, z), E^{0}(x, y, z)$. Expand their into Fourier series on full systems of mutually orthogonal vector functions $H_{m}, E_{m}$ :

$$
H^{0}(x, y, z)=\sum_{m} c_{m} H_{m}(x, y, z), \quad E^{0}(x, y, z)=\sum_{m} d_{m} E_{m}(x, y, z) .
$$

Then the general solution of problem about fading of variable electromagnetic field may be written in the form

$$
\begin{aligned}
& H(x, y, z, t)=\sum_{m} c_{m} \mathrm{e}^{-\gamma_{m} t} H_{m}(x, y, z), \\
& E(x, y, z, t)=\sum_{m} d_{m} \mathrm{e}^{-\delta_{m} t} E_{m}(x, y, z),
\end{aligned}
$$

where $c_{m}$ and $d_{m}$ are some numerical coefficients.

\section{Calculation of Electromagnetic Fields}

At next stage of electromagnetic modelling, we include quasistationary monochromatic external field $\left(H_{0}, E_{0}\right)$ with frequency $\omega$ and internal field on charges $\left(H_{*}, E_{*}\right)$ counting that as a result of controlled chain nuclear reaction into toroidal generator there is established process of fission (the certain finite number $K$ of charged particles and charged splinters of fission and $N$ of neutrons). Calculate suitable fields.

At first we calculate the field $\left(H_{0}, E_{0}\right)$. In quasistationary case the dependence of every value from the time given by means of the factor $e^{-i \omega t}$. Then transforming Maxwell's Equation (7) assumes the form

$$
\Delta H_{0}+k^{2} H_{0}=0,\left.\quad H_{0}\right|_{\Gamma} \in \Omega,
$$

where $k^{2}=\left(\sigma i \omega+\varepsilon \omega^{2}\right) \mu_{0}$. We can obtain $\operatorname{div} E_{0}=0$ from the equation $\operatorname{rot} H_{0}=j_{0}$ ignoring displacement current in investigated quasistationary field and using Equation (8) we can write for $E_{0}$ :

$$
\Delta E_{0}+k^{2} E_{0}=0,\left.\quad E_{0}\right|_{\Gamma} \in \Omega .
$$

Wave Equations ((13), (14)) lead to general Bessel's equations. In our study is considered that toroidal nuclear generator is closed circular solenoid with cylindrical conductive surface on which flows the surface current of conductivity. For such hollow conductor solutions of Equations ((13), (14)) yield known surface skin-effect [4] on Bessel's functions for corresponding equations.

We agree in this time that the current into external winding is direct and has a value $I_{0}$. In this case we can write

$$
\begin{aligned}
& \operatorname{rot} E_{0}=0, \quad j_{0}=\sigma E_{0}, \quad \operatorname{div} j_{0}=0, \\
& \operatorname{rot} H_{0}=j_{0}, \quad \operatorname{div} B_{0}=0, \quad B_{0}=\mu_{0} H_{0},
\end{aligned}
$$

from which, specifically, the existence of constant electric field $E_{0}$ and its potentiality follows inside of conductor. At condition that $\operatorname{div} E_{0}=0$ follows the harmonicity of potential electric field. 
For solution of second group of Equations (15) as usual is introduced the vector potential $A$ so that $B_{0}=\operatorname{rot} A, \operatorname{div} A=0$. When $B_{0}=\mu_{0} H_{0}$ we have

$$
\operatorname{rot}\left(\frac{1}{\mu_{0}} \operatorname{rot} A\right)=j_{0} \text {. }
$$

Then taking into account the identity (5) write for $A$ the Poisson equation: $\Delta A=-\mu_{0} j_{0}$, from which we find

$$
A=\frac{\mu_{0}}{4 \pi} \int \frac{j_{0}}{r} \mathrm{~d} V, \quad H_{0}=\frac{1}{4 \pi} \int \frac{j_{0} \times r}{r^{3}} \mathrm{~d} V,
$$

where $r$ is distance from observation point to volume element $\mathrm{d} V ; r$ in numerator of second formula (16) is radius-vector, incidentally this formula expresses the law of Biot-Savart. Use relations (16) and calculate the field $H_{0}$ for toroid on which direct current $I_{0}$ flows in cylindrical coordinates $\varphi, r, z$. Magnetic field outside of toroidal solenoid $H_{0}=0$ And inside

$$
H_{0 r}=H_{0 z}=0, \quad H_{o \varphi}=\frac{n I_{0}}{2 \pi r},
$$

where $n$ is number of wire spirals.

Now proceed to the calculation of field $\left(H_{*}, E_{*}\right)$ supposed that this field presents the action result of totality of internal charged currents (2) in neglect of their action between itself where $K$ is constant number. Certainly, here the constancy of $K$ and some other values is understood in enough rough and relative sense. Suppose $I_{*}$ is full current on $K$ moving charges in vacuum:

$$
I_{*}=\sum_{i=1}^{K} I_{*_{i}}, \quad \int I_{* i} \mathrm{~d} l_{i}=Z_{i} v_{i},
$$

i.e. moving with velocity $v_{i}$ the charge $Z_{i}$ is equivalent in accordance with expressions (17) to linear current $I_{* i}$ is induced by $i$-th particle. Further, we have taking into account relation (2): $E_{*}=j_{*} / \sigma$. Particles with different on sign charges, as was said earlier, move in opposite directions along of nuclear generator on weakly twisted spirals under the influence of increasing resultant field $(H, E)$.

It is easily establish the direction of magnetic induction vector $B_{* i}$ in observation point which is tangent to magnetic induction lines created by spot charge $Z_{i}$ in vacuum:

$$
B_{* i}=\frac{\mu_{0} Z_{i}}{4 \pi} \frac{v_{i} \times r_{i}}{r_{i}^{3}} .
$$

In the numerator of formula (18) $v_{i}, r_{i}$ are vectors, moreover $v_{i}$ is velocity vector of the point with charge $Z_{i}$ and $r_{i}$ is radius-vector from this point to the observation point. Hence we obtain the expression for the module of vector $B_{* i}:$

$$
\left|B_{* i}\right|=\frac{\mu_{0} Z_{i}}{4 \pi} \frac{\left|v_{i}\right| \sin \alpha}{r_{i}^{2}},
$$

where $\alpha$ is the angle between of vectors $v_{i}$ and $r_{i}$. Note also the 
unidirectionality of vectors $B_{* i}$ and $B_{* j}$ for $i$-th and $j$-th particles if charges $Z_{i}$ and $Z_{j}$ have different signs and opposite directions of motion. From this important circumstance in accordance with the superposition principle follows the relation

$$
B_{*}=\sum_{i=1}^{K} B_{* i} .
$$

Then we determine $H_{*}=B_{*} / \mu_{0}$ due to value $B_{*}$ (19).

\section{Conclusions}

In this article, some questions of mathematical modelling of drastic directional electromagnetic fields generation on toroid as a result of nuclear chain fission process find own reflection. Calculation of electromagnetic field characteristics in external winding of the toroid for various regimes of nuclegen work is produced. On the base presented here general mathematical model of the nuclear electromagnetic generator functioning may be constructed the model of nuclear kinetic process for charged particles and charged splinters into the nuclegen. Besides, may be created the model of neutron kinetics for the nuclegen also. That's exactly contained the significance and the novelty of produced results for the future purpose of the study.

Items [1] [4] are published in Russian except [2] [3].

\section{References}

[1] Tertychny-Dauri, V.Yu. (2008) Galamech. Vol. 3, Hyperreactive Mechanics, Fizmatlit, Moscow.

[2] Tertychny-Dauri, V.Yu. (2015) Effect of $\beta$-Charged Eradiation and Its Calculation in the Nuclear Electrodynamics Theory. IV International Conference on Mathematical Modeling in Physical Sciences, Mykonos, 5-8 June 2015, IOP Journal of Physics: Conference Series, 633, 012037.

[3] Tertychny-Dauri, V.Yu. (2017) Solution of Nuclear Electrodynamics Equations Taking into Account $\beta$-Charged Eradiation. Open Access Library Journal, 4, e3794.

[4] Landau, L.D. and Lifshitz, E.M. (1982) Theoretical Physics. Vol. 8, Electrodynamics of Continuous Media, Nauka, Moscow. 